\title{
NOVEL INTERCONNECTION TECHNOLOGIES FOR INTEGRATED MICROFLUIDIC SYSTEMS
}

\author{
D. Jaeggi ${ }^{1,2}$, B. L. Gray ${ }^{2,3}$, N. J. Mourlas ${ }^{1,2}$, B. P. van Drieënhuizen ${ }^{2}$, \\ K. R. Williams ${ }^{2}$, N. I. Maluf ${ }^{1,2}$, and G. T. A. Kovacs ${ }^{1}$ \\ ${ }^{1}$ Stanford University, Center for Integrated Systems, CISX 218, Stanford, CA 94305-4075 \\ ${ }^{2}$ Lucas NovaSensor, 1055 Mission Court, Fremont, CA 94539 \\ ${ }^{3}$ University of Califomia, Micro Instruments and Systems Laboratory, Davis, CA 95616
}

\begin{abstract}
A new approach to realize silicon based integrated microfluidic systems is presented. By using a combination of silicon fusion bonding (SFB) and deep reactive ion etching (DRIE) $[1,2]$, multi-level fluidic "circuit boards" are fabricated and used to integrate microfluidic components into a hybrid system. As application examples, we demonstrate a multi-level laminating mixer and a multi-level manifold with multiple pressure sensors. To interface the microfluidic system to the macroscopic world, three types of DRIE-fabricated, tight-fitting fluidic couplers for standard capillary tubes are described. One type of coupler is designed for minimal dead space, while another type reduces the risk of blocking capillaries with adhesive. A third design demonstrates for the first time a silicon/plastic coupler combining the DRIE coupler technology with injection-molded press fittings.
\end{abstract}

\section{INTRODUCTION}

Integrated microfluidic systems have recently been gaining interest for many applications including chemical, medical, automotive, and industrial. A major reason is the need for accurate, reliable, and cost-effective liquid and gas handling systems with increasing complexity and reduced size. While a wide range of integrated fluidic devices such as valves, pumps, mixers, and flow sensors have been demonstrated [3], efficient interconnections between these devices and coupling to the macroscopic world are not yet available. However, it is primarily these and other packaging issues that will determine the success of microfluidic devices in commercial applications.

Interconnection schemes for microfluidic devices are analogous to printed circuit boards for electronic parts. A miniature hybrid fluid circuit board based on bonding of plastic has been demonstrated [4]. Microfluidic systems with a higher integration level have used epoxy printed circuit board technology [5] or gasketed stacked modules [6]. A further reduction in size can be obtained with fully monolithic systems. However, the integration complexity of these systems is usually limited by wet silicon etch processes for the definition of channels and vias. The new approach presented in this paper is to use DRIE technology to fabricate complex fluidic "circuit boards". DRIE offers many advantages over wet silicon etch processes, in particular, higher density of fluidic interconnects, precise via holes with uniform cross-sections through the wafer thickness, and the ability to fabricate vias and channels of arbitrary size and shape. The fabrication process includes a combination of DRIE, SFB, and anodic bonding to obtain multi-level fluidic substrates. These fluid circuit boards can be used to build arbitrarily shaped multi-level structures or can be combined with surfacemounted microfluidic devices.
Another major issue in integrated microfluidic systems is the coupling of the fluidic circuits to the macroscopic world. Fluidic couplers consisting of a capillary tube glued into an insertion channel which is iso- or anisotropically etched into a silicon substrate have been previously demonstrated $[\mathbf{7}, \mathbf{8}]$. These couplers do not have accurately fitted insertion channels resulting in difficult handling and increased dead space. Our approach is to use successive DRIE steps to fabricate accurately sized cross-sections for the connecting capillaries. To circumvent the necessity of gluing the capillaries, a new coupler combining DRIE with injection-molded press fittings has been developed allowing the capillaries to be exchanged.

\section{FABRICATION}

Figure 1 illustrates the fabrication process for multi-level fluidic circuit boards. The first deep etch, approximately $100 \mu \mathrm{m}$ in depth,

a)

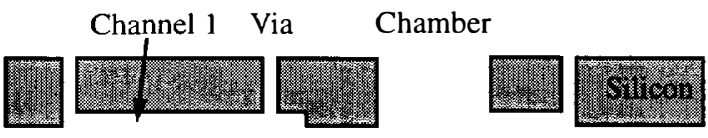

b)

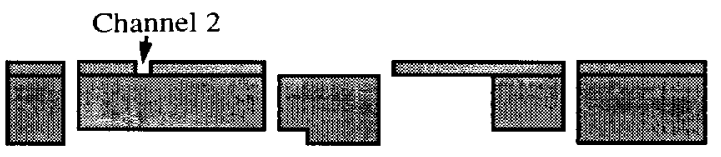

c)

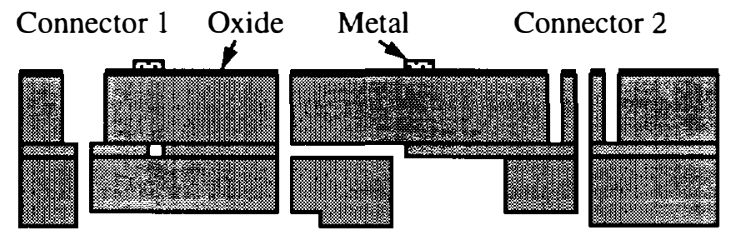

d) Capillary Pressure Sensor

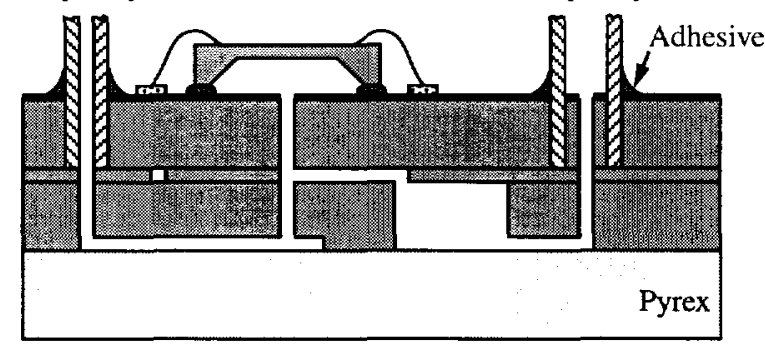

Figure 1. Illustration of the fabrication process for silicon fluidic circuit boards: a) DRIE first channels, through-wafer vias, and chambers; b) SFB, grind back, and polish second wafer and DRIE second channels; c) SFB third wafer, define metal interconnects, and DRIE connectors as well as pressure sensor vias; $d$ ) anodically bond pyrex and attach capillaries and pressure sensors. 
is performed on a double-sided polished silicon wafer to define the first channels. This is followed by a second DRIE step from the opposite side of the wafer to form interconnection vias, 50 $100 \mu \mathrm{m}$ in diameter, all the way through the $390 \mu \mathrm{m}$ thick wafer. All silicon etches are performed using a tool from Silicon Technology Systems (Abercarn, U.K.) at etch rates of $2-3.2 \mu \mathrm{m} / \mathrm{min}$. A second silicon wafer is bonded to the first and polished down to $100 \mu \mathrm{m}$ in thickness. Second level channels are then etched through the second wafer. A third SFB step caps the second level of channels with a third $390 \mu \mathrm{m}$ thick wafer. Aluminum metallization $(0.8 \mu \mathrm{m})$ is patterned on top of the three-wafer stack to provide electrical interconnects to the surface mounted components, e.g., pressure sensors or microvalves. It is isolated from the silicon by $0.3-0.6 \mu \mathrm{m}$ of thermally grown silicon oxide. The final DRIE step etches entirely through the top wafer, defining couplers to capillary tubing and access holes for surface-mounted fluidic components. A transparent glass substrate that is anodically bonded to the bottom of the stack aids in flow visualization, but may be replaced by a silicon wafer to result in an all silicon stack.

a)

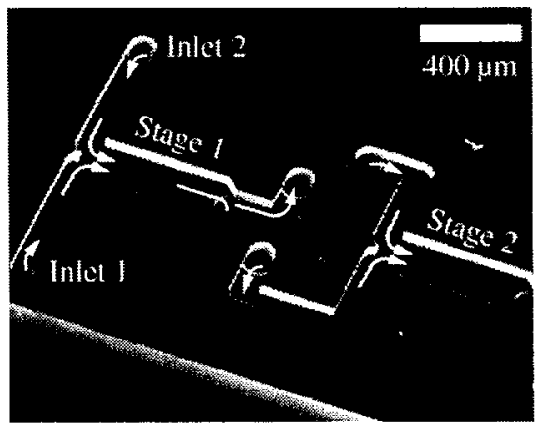

b)

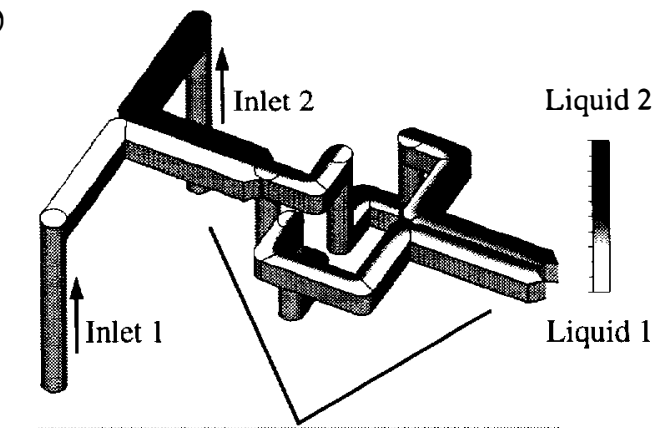

c)

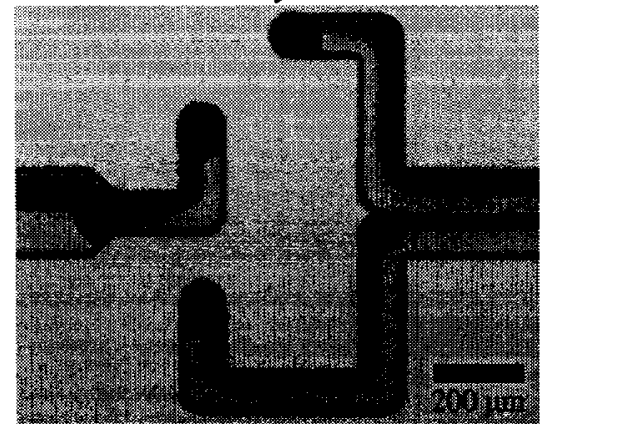

Figure 2. Illustrations of a multi-level laminating mixer. Mixing occurs by successive separation and reuniting of fluid streams to increase the contact area between the two fluids, resulting in faster mixing by diffusion: a) SEM photograph of first channel level and vias of first two (of six) stages; b) CFD simulation result; c) Photograph of an actual mixer operating at $60 \mu \mathrm{l} / \mathrm{min}$ using water dyed with different colors.
The result of the overall process is a four-wafer stack, with two interconnected channel levels that can be accessed by surface mount components and external capillaries through vias etched in the top wafer.

\section{MULTI-LEVEL STRUCTURES}

The process shown in Figure 1 can be used to fabricate structures that utilize three-dimensional flow patterns for operation. An example of such a structure is a multi-level laminating mixer [9]. Mixing of two fluids in a reasonable amount of time is fundamental to the creation of "on-chip" microfluidic processing systems. However, the channel sizes and flow rates associated with such processing systems usually imply low Reynolds numbers, precluding turbulence as a mixing mechanism. Mixing by diffusion can be very slow unless the two fluids are laminated to increase their contact area.

Figure 2a shows an SEM photograph of one level of channels and vias of the first two (of six) stages of a laminating mixer. The channels are $100 \mu \mathrm{m}$ deep. Two fluids entering the inlet ports laminate at the first horizontal junction, producing two side-by-side fluid streams. Successive vertical separation and horizontal reuniting of fluid streams increases the number of laminates with each stage and, thus, the contact area between the two fluids [9]. After the second stage, four laminates exist, with each following stage doubling the number of laminates to $2^{n}$, where $n$ is the stage number. We performed computational fluid dynamics (CFD) simulations using the tools of CFD Research Corporation (Huntsville, AL) [10]. Figure $2 \mathrm{~b}$ shows a simulation result of the first two stages of the mixer, where the differently dyed water at each inlet port has a flow rate of $30 \mu \mathrm{l} / \mathrm{min}$. The Schmidt number used was 1200 , evaluated from separate measurements. Figure $2 \mathrm{c}$ shows a close-up of the first vertical split and second reunification in a fabricated mixer with the same input conditions. We see the expected four laminates in the second stage, which is in qualitative agreement with simulation results.

\section{HYBRID SYSTEMS}

As a demonstration of an integrated hybrid microfluidic system, five pressure sensors were directly mounted on a silicon substrate

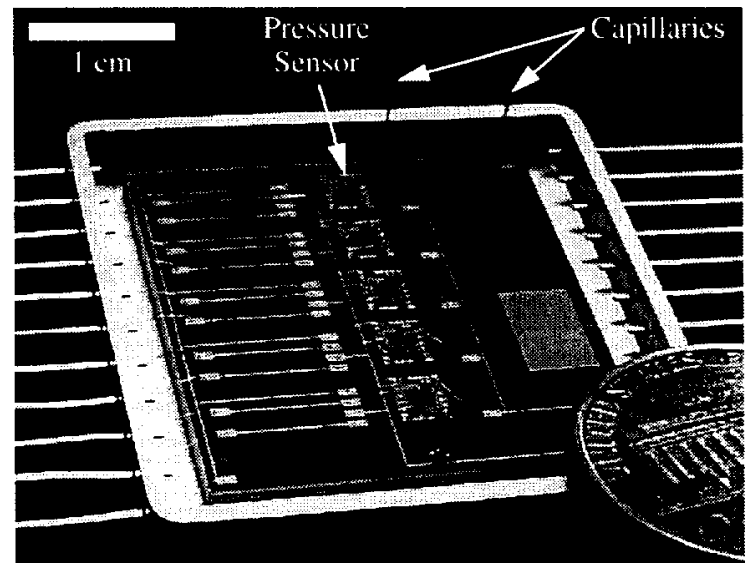

Figure 3. Photograph of a fluidic circuit board demonstration system with five pressure sensors mounted on a substrate with an embedded microchannel. The five pressure sensors are mounted $4 \mathrm{~mm}$ apart over $100 \mu \mathrm{m}$ diameter access holes to the buried 100- $\mu$ m square channel. 


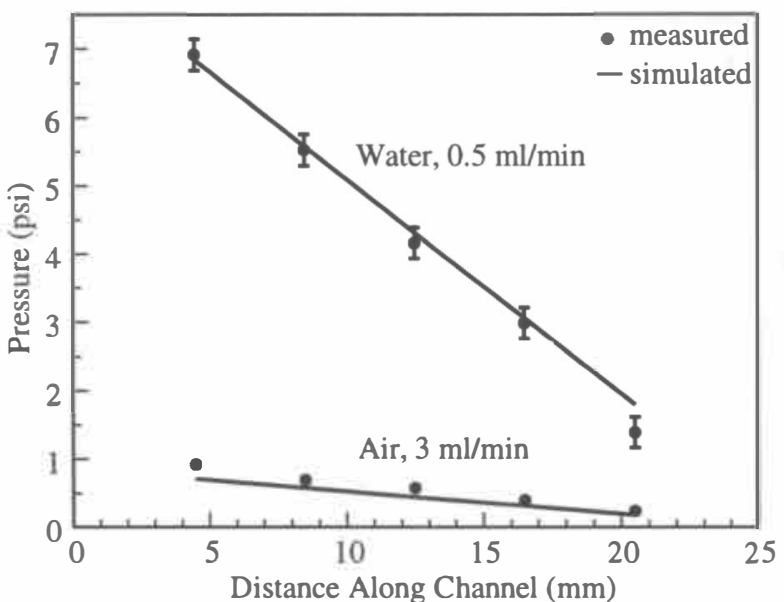

Figure 4. Measured and simulated data for air and water showing

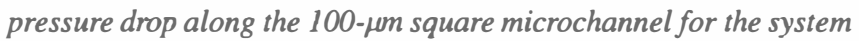
shown in Figure 3. The simulation results were obtained using CFD simulations.

with an embedded $100-\mu \mathrm{m}$ square microchannel and pressure ports leading from the channel to the sensors (Figure 3). The commercially available piezoresistive pressure sensors (Lucas NovaSensor, Fremont, CA) have a pressure range of 0 - 30 psi. Sensitivities, calibrated after surface mounting using a dead-weight tester, measured $4.7-5 \mathrm{mV} / \mathrm{psi}$. A syringe pump was used to provide constant flow rates. Pressure inside the channel was measured for water and air flowing at $500 \mu \mathrm{l} / \mathrm{min}$ and $3 \mathrm{ml} / \mathrm{min}$, respectively (Figure 4). The worst case error between measured data and simulation results obtained using CFD modeling was $25 \%$. The kinematic viscosities used in the models were $1 \times 10^{-6} \mathrm{~m}^{2} / \mathrm{s}$ and $1.51 \times 10^{-5} \mathrm{~m}^{2} / \mathrm{s}$ for water and air, respectively.

\section{FLUIDIC COUPLERS}

Fluidic couplers for standard capillary tubing are fabricated using the process sequence shown in Figure 1. The left coupler in Figure 1d illustrates the first design. DRIE is used to define circular holes matching the inside and outside diameter of the capillaries,

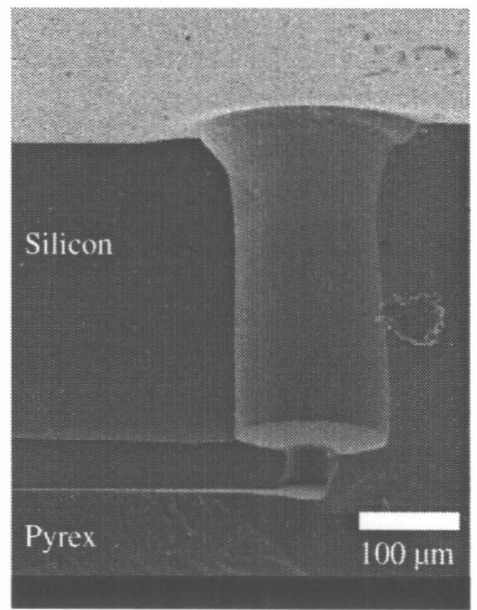

Figure 5. SEM photograph of a minimum dead space fluidic coupler for capillaries with $150 \mu \mathrm{m}$ and $50 \mu \mathrm{m}$ outer and inner diameters, respectively.

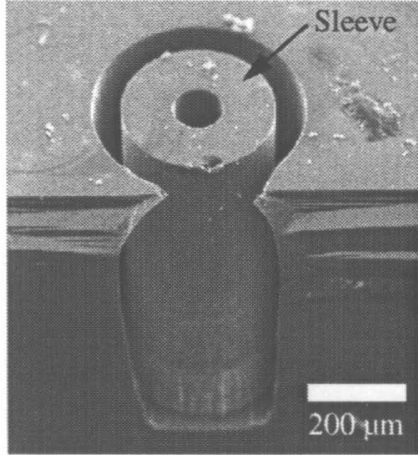

a)

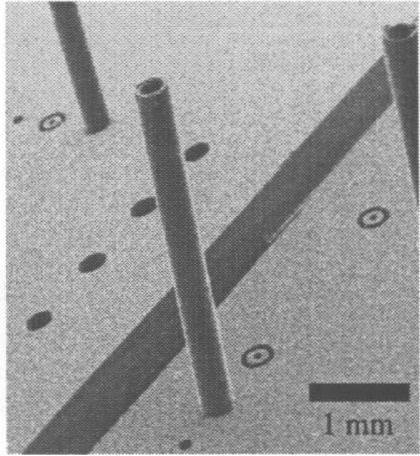

b)
Figure 6. a) SEM photograph of a fluidic coupler with a $110 \mu \mathrm{m}$ thick sleeve around the bore to prevent blocking of capillaries with adhesive. The silicon is partially cleaved to show the sleeve; b) SEM photograph of capillaries inserted into the sleeve coupler.

thus, eliminating any dead space. Figure 5 shows a SEM photograph of the minimum dead space coupler. The capillary is inserted into the opening and held with adhesive.

It was observed that improperly cleaved capillaries with a cusp at the end did not fit well into the insertion channel and allowed seeping of adhesive into the capillary/silicon interface. Therefore, the second type of coupler has a silicon "sleeve" around the etched bore, as illustrated in the right coupler in Figure 1d. Figure 6a shows a SEM photograph of a coupler with a $110 \mu \mathrm{m}$ thick sleeve. The sleeve acts as barrier to adhesive and enhances the mechanical integrity of the coupling, but also introduces some dead volume. Figure $6 \mathrm{~b}$ shows fused silica capillaries with $250 \mu \mathrm{m}$ and $360 \mu \mathrm{m}$ inner and outer diameters, respectively, inserted into the sleeve couplers. To quantify the dead volume introduced, CFD simulations were performed. A dead volume on the order of $2 \mathrm{nl}$ at a flow rate of $100 \mu \mathrm{l} / \mathrm{min}$ was estimated for the structure shown in Figure 6a.

Figure 7 is an illustration of the third type of coupler which requires no gluing of capillaries. The plastic press fitting is fabricated using injection molding of polyoxymethylene (POM) plastic [11]. To align and hold the plastic part in place, the silicon stack and the pyrex have additional through holes for pegs fabricated by DRIE and ultrasonic drilling, respectively. The plastic pegs are

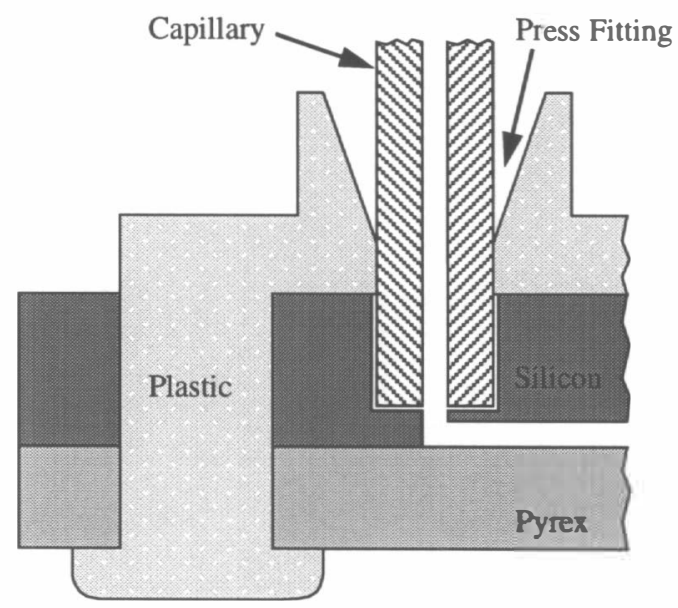

Figure 7. Illustration of siliconplastic coupler with press fitting. Heat-staked pegs are used to hold the injection molded plastic part against the silicon. 


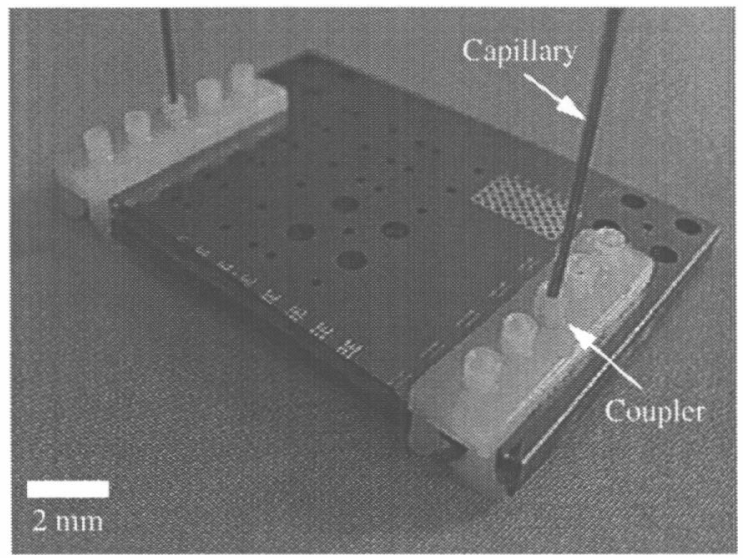

Figure 8. Photograph of two arrays of silicon/plastic couplers with inserted capillaries accessing a 50- $\mu \mathrm{m}$ square microchannel.

melted with a heat-staking tool at temperatures reaching $250^{\circ} \mathrm{C}$. The tool consists of an adapted soldering-iron with a copper block attached to it. Figure 8 shows two arrays of couplers with inserted capillaries that are connected through a microchannel in the substrate. Leakage tests of the new couplers were performed by pressurizing a blocked microchannel with water and measuring the pressure loss versus time. Figure 9 shows that the new silicon/plastic coupler alone is not leak proof at a pressure of 60 psi. By introducing a silicone gasket, cut from a $500 \mu \mathrm{m}$ thick sheet, between the plastic and the silicon, we obtained a sealed fluidic coupler system for pressures up to a maximum of $60 \mathrm{psi}$. This compares to glued capillary connectors which are known to hold pressures up to approximately 2000 psi [8]. To quantify the mechanical integrity of the silicon/plastic couplers, pull-tests with a load cell were performed. We measured pull-out forces up to $2 \mathrm{~N}$.

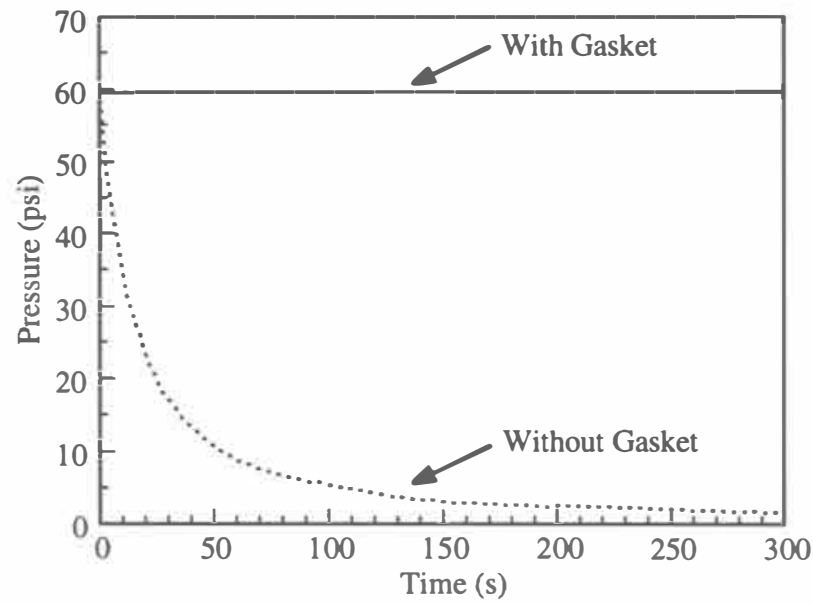

Figure 9. Measured water pressure loss in a blocked test channel indicating a sealed connection for a silicon/plastic coupler with a $500 \mu \mathrm{m}$ thick silicone gasket.

\section{CONCLUSIONS}

We have presented a novel technology for the fabrication of fluidic circuit boards including couplers to standard capillary tubes. The circuit board can either be used to interconnect hybrid mounted flu- idic devices such as pressure sensors or to obtain multi-level fluidic structures such as laminating mixers. A novel silicon/plastic press fitting coupler is user-connectable to capillary tubing and is leak proof for pressures below 60 psi. For higher pressures, adhesiveheld capillaries may be necessary.

\section{AKNOWLEDGEMENTS}

Funding for this work was provided by the DARPA MicroFlumes Program (Contract Number: N66001-96-C-8631). The authors would like to thank R. Scimeca of Lucas NovasSensor, Fremont, CA, M. G. Giridharan of CFD Research Corporation, Huntsville, AL and T. Callenbach of H. Weidmann AG, Plastic-Technologies, Rapperswil, Switzerland for their help and technical discussion with processing, CFD modeling, and heat-staking, respectively. Load cell measurements by G. Cornella, Department of Materials Science and Engineering, Stanford University, CA are gratefully acknowledged.

\section{REFERENCES}

1. E. H. Klaassen, K. Petersen, J. M. Noworolski, J. Logan, N. I. Maluf, J. Brown, C. Storment, W. McCulley, and G. T. A. Kovacs, "Silicon Fusion Bonding and Deep Reactive Ion Etching; A new Technology for Microstructures," Transducers '95 Digest of Technical Papers, vol. 1, Stockholm, 1995, pp. 556-559.

2. B. P. van Drieënhuizen, N. I. Maluf, I. E. Opris, and G. T. A. Kovacs, "Forced-Balanced Accelerometer with mG Resolution, Fabricated using Silicon Fusion Bonding and Deep Reactive Ion Etching," Transducers '97 Digest of Technical Papers, vol. 2, Chicago, 1997, pp. 1229-1230.

3. P. Gravesen, J. Branebjerg, and O. S. Jensen, "Microfluidics - A Review,” J. Micromech. Microeng., vol. 3, 1993, pp. 168-182.

4. D. Verlee, A. Alcock, G. Clark, T. M. Huang, S. Kantor, T. Nemcek, J. Norlie, J. Pan, F. Walsworth, and S. T. Wong, "Fluid Circuit Technology: Integrated Interconnect Technology for Miniature Fluidic Devices," Proc. of the Solid-State Sensor and Actuator Workshop 96, Hilton Head, 1996, pp. 9-14.

5. T. S. J. Lammerink, V. L. Spiering, M. Elwenspoek, J. H. J. Fluitman, and A. van den Berg, "Modular Concept for Fluid Handling Systems: A Demonstrator Micro Analysis System," Proc. MEMS '96, San Diego, 1996, pp. 389-394.

6. W. K. Schomburg, B. Büstgens, J. Fahrenberg, and D. Maas, "Components for Microfluidic Handling Modules," Micro Total Analysis Systems, ed. by A. van den Berg and P. Bergveld, Kluwer Academic Publishers, Dordrecht, 1995, pp. 255-258.

7. R. J. Reay, R. Dadoo, C. W. Storment, R. N. Zare, and G. T. A. Kovacs, "Microfabricated Electrochemical Detector for Capillary Electrophoresis," Proc. of the Solid-State Sensor and Actuator Workshop 94, Hilton Head, 1994, pp. 61-64.

8. V. L. Spiering, J. N. van der Moolen, G.-J. Burger, and A. van den Berg, "Novel Microstructures and Technologies Applied in Chemical Analysis Techniques," Transducers ' 97 Digest of Technical Papers, vol. 1, Chicago, 1997, pp. 511-514.

9. H. Möbius, W. Ehrfeld, V. Hessel, T. Richter, "Sensor Controlled Processes in Chemical Microreactors," Transducers '95 Digest of Technical Papers, vol. 1, Stockholm, 1995, pp. 775-778.

10. CFD-GEOM, CFD-ACE, CFD-VIEW Manuals, CFD Research Corporation, Huntsville, 1997.

11. Concise Encyclopedia of Polymer Science and Engineering, ed. by J. I. Kroschwitz, John Wiley \& Sons, New York, 1990. 